\title{
Deux maisons, égales en dignité
}

Une approche anthropologique des déterminants du développement durable en contexte amazonien " traditionnel »

\section{Florent Kohler, Anna Greissing et François-Michel Le Tourneau}

\section{(2) OpenEdition}

Journals

Édition électronique

URL : http://journals.openedition.org/developpementdurable/8173

DOI : 10.4000/developpementdurable.8173

ISSN : 1772-9971

Éditeur

Association DD\&T

Référence électronique

Florent Kohler, Anna Greissing et François-Michel Le Tourneau, «Deux maisons, égales en dignité », Développement durable et territoires [En ligne], Varia (2004-2010), mis en ligne le 30 septembre 2010, consulté le 10 décembre 2020. URL : http://journals.openedition.org/developpementdurable/8173 DOI : https://doi.org/10.4000/developpementdurable.8173

Ce document a été généré automatiquement le 10 décembre 2020.

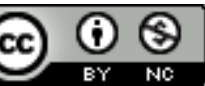

Développement Durable et Territoires est mis à disposition selon les termes de la licence Creative Commons Attribution - Pas d'Utilisation Commerciale 4.0 International. 


\title{
Deux maisons, égales en dignité
}

Une approche anthropologique des déterminants du développement durable en contexte amazonien « traditionnel »

\author{
Florent Kohler, Anna Greissing et François-Michel Le Tourneau
}

1 L'Amazonie brésilienne est devenue un laboratoire mondial du meilleur et du pire. Le pire : déforestation incontrôlée, projets démesurés d'infrastructures sans étude d'impact. Le meilleur: une multitude de sites d'expérimentation visant à mettre en place des alternatives de subsistance pour les communautés locales, "riveraines» (qui représentent environ cinq millions d'individus éparpillés sur environ quatre millions de $\mathrm{km}^{2}$ ) dont nous présentons ici deux exemples. Nous verrons comment les interventions extérieures, la multiplication des interlocuteurs, tous animés des meilleures intentions, ébranlent la cohésion communautaire, forgée au long de décennies par la mise en place d'un contrôle social souvent difficile à appréhender ${ }^{1}$.

2 Situées l'une au nord-est, l'autre au sud-ouest de l'Amapá, Vila Velha do Cassiporé et Vila de São Francisco do Iratapuru pourraient sembler à première vue des communautés jumelles : 300 habitants pour la première, un peu moins de 200 pour la seconde. Toutes deux sont isolées, n'ayant pas d'accès à la route, et ont connu, au long de leur histoire, de brusques changements d'activités et des saignées de population, jusqu'à parvenir, dans les années 1990, à établir des alliances avec des organismes publics, respectivement l'INCRA (Institut National de la Colonisation et de la Réforme Agraire) et la SEMA (Secrétariat à l'environnement de l'Etat d'Amapá). Ces alliances s'imposèrent à la suite de l'effondrement des réseaux locaux de commercialisation, accompagné de restrictions imposées par les nouveaux maitres de la région: l'IBAMA (Institut Brésilien de l'Environnement) qui gère le Parc National du Cap Orange à l'embouchure du Cassiporé, et l'entreprise Jari Celulose sur le Jari, où débouche la rivière Iratapuru. On discerne donc, à travers les choix privilégiés par ces communautés en état de «stress socioéconomique", un jeu d'acteurs institutionnels poursuivant des logiques discordantes, dont les populations locales ont appris à tirer parti. Ces alliances ne constituent cependant pas une panacée, car c'est d'abord leurs ressources propres et les dynamiques sociales à l'œuvre dans ces communautés qui conditionnent le succès ou l'échec des projets mis en place par ces puissants alliés. Il nous faudra donc nous 
interroger sur ce qui fait communauté $e_{2}$ au sens proposé par Max Weber (1922), qui définit la communalisation comme étant fondée sur la croyance subjective en une origine commune, une histoire, des traditions et une culture partagées.

3 Le premier objectif de cette recherche est de déceler, par la comparaison de deux communautés similaires, ce qui fait qu'une dynamique collective peut, ou non, se mettre en place et se perpétuer. De ce point de vue, si les propositions de Max Weber conservent toute leur validité, on peut suggérer que cette destinée collective peut trébucher lorsque la diversité des origines, la multiplication des opportunités économiques et donc des intervenants extérieurs ébranle la cohésion de la communauté. Notre deuxième objectif, dans le cadre du projet DURAMAZ dont dérive cet article, est de démontrer la complémentarité des approches anthropologique et socioéconomique: si cette dernière (Greissing, Kohler, Le Tourneau \& Picanço, 2008) peut éclairer le comment, une approche anthropologique fondée sur l'observation participante et des entretiens non dirigés peut quant à elle éclairer le pourquoi du succès ou de l'échec de projets communautaires.

Notre article, après une première partie consacrée à la communauté de Vila Velha, où les projets peinent à s'enraciner, proposera en deuxième partie, et grâce à cet éclairage, d'examiner les raisons du succès actuel de la communauté d'Iratapuru. Nous nous attacherons en conclusion à relever et à résoudre le paradoxe inhérent aux projets de développement durable destinés aux communautés traditionnelles: si le discours écologique ou le compromis avec le développement durable repose sur des pratiques, comment concilier l'amélioration du niveau de vie (et les nouveaux besoins qui en découlent) avec le maintien d'activités traditionnelles à faible valeur ajoutée sans modifier, justement, le mode de vie et de production? C'est la viabilité même du système extractiviste ${ }^{2}$ qui est en jeu dans la résolution de ce paradoxe, inscrit dans la loi brésilienne (Loi $\mathrm{n}^{\circ}$ 9.985/2000, créant le "Système National d'Unités de Conservation ", article 28, paragraphe unique) qui détermine que «les Unités de Conservation [d'usage direct] assurent aux populations traditionnelles la préservation de leur mode de vie, $\mathrm{y}$ compris en ce qui touche à l'exploitation des ressources naturelles. »

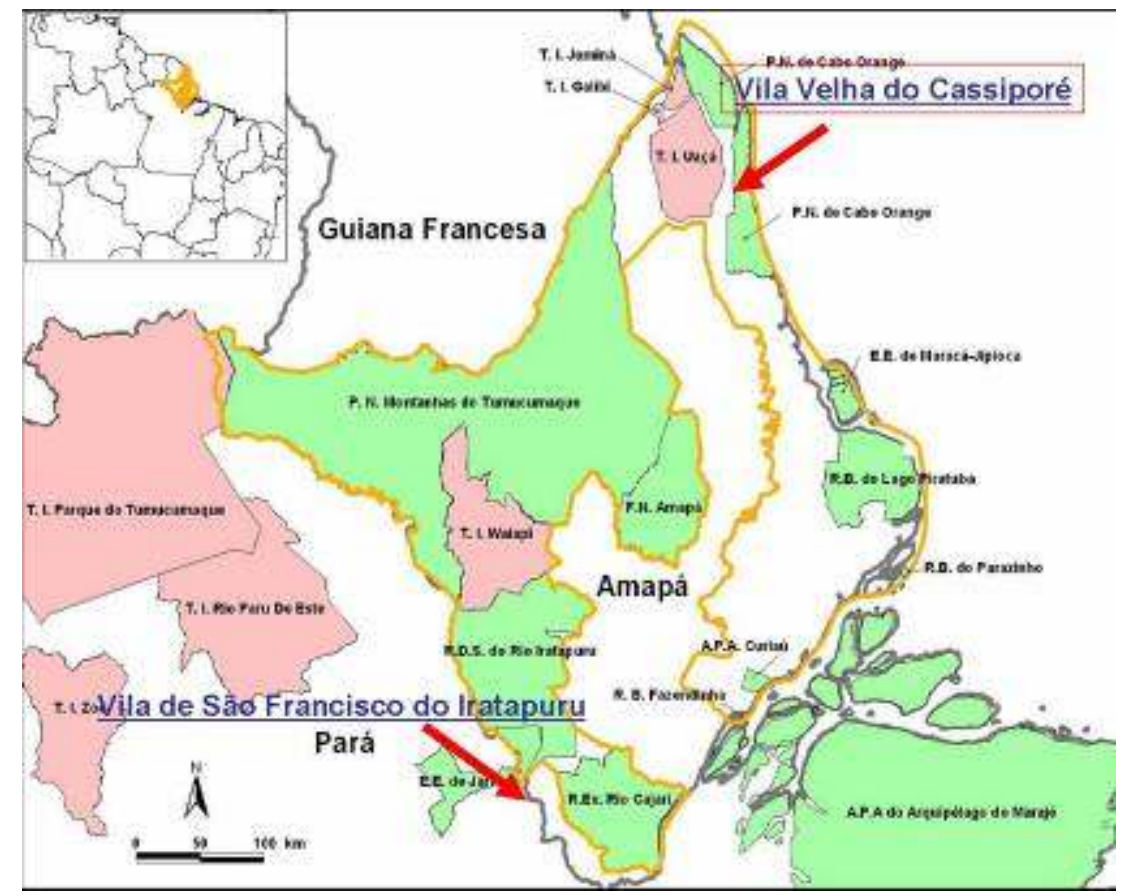




\section{Vila Velha de São José (Fleuve Cassiporé)}

Le nord de l'Amapá, ancienne zone de conflit frontalier franco-brésilien, représente une forte interrogation en termes historiques, pour être resté, durant plus de trois siècles, une zone de passage avec relativement peu d'implantations permanentes. Selon Pierre et Françoise Grenand (1987), si l'on excepte les Palikur, toujours présents le long de l'Urucauá, plus de vingt-cinq ethnies passèrent ou trouvèrent refuge dans les bassins hydrographiques des fleuves Oyapock, Uaçá et Cassiporé, fuyant la colonisation, les chasseurs d'esclaves et les guerres interethniques. Ces ethnies disparurent ou, leur faiblesse démographique étant devenue critique, subirent des recompositions culturelles, comme ce fut le cas probable de ceux que l'on appelle aujourd'hui les Galibi-Marworno installés le long de l'Uaçá. Tant l'Uaçá que le Cassiporé connurent l'implantation épisodique de non-Indiens. Le profil de cette immigration diffère beaucoup d'un siècle à l'autre. Durant le XIX et jusqu'au début du XXe siècle, les migrants venaient du Nord, de la Guyane française et même du Surinam. Cette immigration varie dans ses motifs. Le plus souvent, toutefois, c'est l'attrait des sites d'orpaillage qui explique ces parcours. Avec la résolution du conflit frontalier en faveur du Brésil, le flux d'immigration issu du sud (Pará et Nordeste) s'accentua. L'orpaillage resta en grande partie entre les mains des Créoles guyanais : les Brésiliens, venus seuls le plus souvent, n'avaient d'autre option que de se fondre dans les populations indigènes, contractant des mariages et fondant des dynasties.

\subsection{Tradition et opportunisme économique}

Alors que nous entrons dans l'histoire contemporaine de ces populations, il nous faut rappeler qu'elles maintenaient avant l'arrivée de la route nationale 156, dans les années 1980, des relations étroites, aussi bien commerciales que culturelles, religieuses et matrimoniales. Kumarumã, Vila Velha et Taperebá formaient en vérité un réseau socioéconomique bien articulé et organisé, avec une ingérence extérieure limitée aux marreteiros (commerçants fluviaux) et aux pêcheurs de Belém (à partir des années 1970), et à l'arrivée épisodique d'immigrants nordestins ou d'orpailleurs guyanais. Le relatif isolement de la région, accessible uniquement par la mer, était dû aux dangers du ressac en ce littoral de hauts-fonds. Les seuls transports réguliers étaient les bateaux chargés de bétail qui approvisionnaient la colonie militaire de Clevelândia do Norte (sur l'Oyapock) ou Cayenne.

7 La rente principale des populations locales provenait des activités d'extraction destinées aux atravessadores (intermédiaires), et surtout en la fourniture de produits et d'aliments aux orpailleurs travaillant en amont du Cassiporé. Les pirogues et la farine de manioc fabriqués par les Indiens de l'Uaçá étaient échangés contre du poisson et des produits manufacturés dans les vilas (bourgades) du Cassiporé, et de là transitaient jusqu'aux sites d'orpaillage. Mais les produits d'extraction, destinés à l'exportation, jouaient également un rôle crucial dans les échanges, la localisation des ressources naturelles conditionnant les déplacements de familles entières: ainsi de l'actuelle Vila de Taperebá, implantée dans les années 1940 du fait de la présence dans les environs d'arbres à latex. Dans les années 1940 à 1950, la chasse prédominante fut celle des peaux de jaguar, d'ocelot, de 
loutre, de pécari, et surtout celles de caïman noir, cette dernière chasse se poursuivant jusque dans les années 1970. Par ailleurs, le Cassiporé connut un sursaut - éphémère - de l'activité d'orpaillage dans les années 1960. Les habitants de la région ne travaillaient pas directement dans l'orpaillage, toujours contrôlé par des Guyanais, mais fournissaient aux orpailleurs tout ce dont ils avaient besoin. La fréquence des bateaux augmenta alors et l'aisance générale également.

8 L'interdiction du commerce de peau, le déclin de l'orpaillage, le tracé de la route nationale 156 qui passa au large de ces communautés, les éloignant ainsi des nouvelles opportunités, sont les raisons les plus évidentes de leur état actuel de marasme économique, malgré leurs ressources en termes d'extractivisme (cacao natif et palmier wassaï - ou açaï, Euterpe oleracea, en particulier). Selon Sebastião Pinheiros, ancien représentant de communauté, la population de Vila Velha, à la fin des années 1960, était tombée à une douzaine de familles. La fin des activités lucratives entraîna le déclin des réseaux traditionnels d'échange entre les deux fleuves. La création du Parc National du Cap Orange, en 1980, qui contrôlait sévèrement l'accès au Cassiporé, et la démarcation de la Terre Indigène Uaçá (processus qui dura de 1975 à 1992), menèrent à une reconfiguration des partenariats habituels. Avec la fin de la marretagem (commerce fluvial) sur le Cassiporé, interdite par l'IBAMA, et la politique de différenciation ethnique (ou « ethnicisation ») dans l'Uaçá, les différentes communautés élaborèrent de nouvelles stratégies d'alliance, chacune optant pour un partenaire institutionnel puissant pour opposer un contrepoids à l'IBAMA et sortir de l'isolement : la FUNAI (Fondation Nationale de l'Indien) pour le village Galibi-Marworno de Kumarumã, l'INCRA pour Vila Velha ${ }^{3}$. C'est donc auprès d'institutions fédérales que les habitants désemparés cherchèrent à retrouver la protection autrefois accordée par les commerçants fluviaux et les intermédiaires, rompant par la même occasion les anciens liens de solidarité et de " compérage » (rapports unissant les parrains d'un enfant à ses parents).

Sebastião Pinheiros joue alors un rôle important, comme représentant de la communauté, en s'efforçant de dynamiser le travail coopératif autour de l'extraction de cacao. Mais « malheureusement, dit-il, l'être humain, principalement le Brésilien, ne connaît pas l'union". L'amertume qui ressort de ces propos naît d'un constat : à de multiples reprises fut tentée la mise en place d'une entreprise agroextractiviste autour du cacao natif, dont un ultime avatar en décembre 2007, lorsqu'est lancé le projet « d'appui à l'activité cacaoyère » de la communauté de Vila Velha do Cassiporé, non par l'INCRA mais par le Secrétariat de l'Etat d'Amapá à l'Industrie, au Commerce et aux Mines (SEICOM) avec l'appui de la RURAP (Institut du Développement Rural - Amapá). Cette initiative, qui vise à renforcer l'association agroextractiviste et à mettre en place l'exploitation rationalisée du cacao (qui jusque-là pourrissait sur pied faute de prix plancher), relève donc de l'Etat d'Amapá, et non du gouvernement fédéral ${ }^{4}$. La multiplication des projets visant à renforcer la dynamique communautaire constitue un aveu d'échec, et contraste avec le relatif succès de l'implantation de l'INCRA, puisque l'attribution de lots pour une exploitation agricole classique semble fonctionner.

\subsection{Les protestants (« crentes »), fléau de Dieu?}

Comme l'explique Sebastião Pinheiros, le principal obstacle à l'union en vue d'une activité collective (coopérative ou syndicat) réside dans la multiplication des Eglises (la catholique dont il est le principal représentant, mais aussi l'Assemblée de Dieu et la Congrégation 
Chrétienne du Brésil, églises évangéliques). De ce fait, «l'Association agroextractiviste du Cassiporé» demeure à ce jour une coquille vide: "Nous avons aujourd'hui les trois religions dans notre communauté, c'est alors qu'a commencé le cauchemar. (...) Nos religions ne travaillent pas unies. (...) Il n'y a pas de disputes, mais on voit qu'ils veulent toujours être les meilleurs. Ils font des fêtes, ils apparaissent aux nôtres, mais en termes d'union ils se prennent toujours pour les meilleurs.»

11 La création d'une Église protestante - et a fortiori, de plusieurs - affecte en profondeur la vie communautaire, par le mépris affiché pour la traditionnelle beuverie de fin de semaine, par le rejet des croyances locales relatives aux esprits, au mauvais œil et autres, et par le refus de participer aux traditionnelles fêtes de saints, moment privilégié de la sociabilité locale voire régionale. C'est ce que Sebastião Pinheiros traduit par l'expression «ils veulent être les meilleurs»; or cette position intransigeante finit par atteindre le résultat désiré : plus fermes dans leur foi et leurs principes, les évangéliques (terme désignant les membres des Églises protestantes) attirent progressivement dans le giron de leurs Églises les autres membres de la communauté. Comme l'observait déjà Max Weber à la fin du XIX ${ }^{e}$ siècle, le succès de la foi protestante est dû à son impact direct sur la qualité de vie de celui qui la pratique : la sociabilité décline pour se limiter à la famille nucléaire, et cette famille se concentre sur les activités productives sans plus souffrir d'interférence de la boisson, des querelles ou des relations extraconjugales. Cette différence est affirmée avec beaucoup d'orgueil par Dona Soeli Martins, une habitante de Vila Velha de la même génération que Sebastião, convertie dès l'âge de quinze ans, qui rejette avec dédain l'idée de marier ses filles à un membre de la communauté, tout comme elle-même s'y refusa : "C'est très bien quand ta fille ou ton fils se marie avec des gens de l'extérieur, parce qu'ici il y en a beaucoup qui sont de la même famille, du même sang. Ma famille, c'est différent, nous sommes les Martins : regarde la différence avec les gens d'ici! (...) Moi qui suis née ici, Sabá (Sebastião Pinheiros) a été mon camarade de classe, mais je n'ai jamais songé à épouser quelqu'un d'ici. »

\subsection{Jeu de perceptions antagonistes}

Un détail intéressant est que Dona Soeli, qui insiste au cours de l'entretien sur l'état d'abandon de la bourgade, finit par faire des herbes folles qui envahissent les ruelles le symbole de la désunion et de l'absence d'autorité des représentants, un discours que chacun reprend à son compte, les représentants eux-mêmes accusant les habitants de ne pas se plier aux travaux d'utilité collective. Nous avons toutefois relevé bien d'autres facteurs de dissensions, parmi lesquels on peut compter la perception radicalement opposée des alliés potentiels: les catholiques évoquent volontiers leurs excellents, quoique déclinants, rapports avec les Indiens (catholiques) de Kumarumã, rapports ayant résulté en quelques mariages. Les évangéliques, quant à eux, expriment des sentiments qui vont jusqu'à la répulsion. Les plus féroces à ce sujet furent un couple âgé, ayant passé deux ans à Kumarumã, leur départ étant motivé par le fait que les Indiens «ensorcelaient les gens par perversité ».

Division dans les croyances, division dans la perception des amis et alliés, division, enfin, quant à la légitimité de l'occupation du territoire. La famille Pinheiros, aujourd'hui majoritaire, est considérée comme invasive (elle est arrivée dans les années 1950) par les habitants plus anciens, ainsi de Procopo de Almeida, 80 ans et catholique, dont le père était Indien du Surinam et la mère Indienne (métissée) du Curipi. Ce caractère invasif est 
motivé par deux raisons; la première est que cette famille n'est pas "prêteuse »; la deuxième (qui n'est que suggérée mais me semble la cause réelle), est que la famille Pinheiros, au moment de son installation, comptait un grand nombre de fils qui accaparèrent les femmes en âge de se marier, concentrant ainsi le capital transmissible. L'accusation d'avarice est d'ailleurs retournée à l'envoyeur par le chef de la famille Pinheiros, Sebastião, dénonçant le peu de solidarité du couple formé par ce même Procopo et son épouse, qui tiennent l'unique commerce de la bourgade: "Ils ont un commerce ici, mais ce qui est vendu un réal à Oiapoque, ils le vendent ici pour quatre réaux. Grâce à Dieu ce sont de braves gens, des fils d'ici, ils nous donnent cet appui, mais c'est un appui ruineux .» (é um apoio tirando).

14 Arrêtons-nous un instant sur l'expression employée par Sebastião Pinheiros : «c'est un appui ruineux»; il emploie une tournure similaire lorsqu'il s'agit de la relation avec l'IBAMA : «Ils nous font plaisir d'un côté et nous plantent un couteau de l'autre, une caresse d'un côté et ils nous poignardent de l'autre, en nous ôtant nos ressources" L'emploi réitéré d'expressions paradoxales nous semble traduire la complexité de la situation de Vila Velha telle qu'elle est ressentie par les habitants eux-mêmes, qui ont intériorisé les conflits et dissensions au point de ne pouvoir émettre de jugement tout d'un bloc. Or il nous semble que l'attitude des habitants de Vila Velha, tiraillés entre INCRA et IBAMA, traduit l'ambivalence de leurs attentes à l'égard des perspectives économiques qui leur sont proposées, et qui dépendent, en fin de compte, de l'adoption d'une éthique communautaire.

\subsection{IBAMA \& INCRA : la dialectique de la préservation et du progrès}

15 La position de la communauté vis-à-vis de l'Institut Brésilien de l'Environnement fut construite en plusieurs étapes, qui suivirent un probable effondrement collectif, un sentiment d'abandon exprimé, ailleurs (à Taperebá, en zone de "cœur de parc»), de manière saisissante : «Jusqu'ici, nous avions toujours cru que le gouvernement nous appuyait ». C'est à la suite de cet ébranlement que fut recherchée l'alliance avec l'INCRA, obtenue en 1999 : grâce à l'élévation au rang « d'agroville » (ce qui implique une révision du cadastre et des facilités bancaires et matérielles), Vila Velha vit revenir 40 familles parmi celles qui l'avaient abandonnée quelques années auparavant. L'attrait exercé est dû à l'implantation d'un enseignement "modulaire» (pas de personnel en poste fixe mais des équipes tournantes), la possibilité d'éduquer ses enfants étant une condition du retour. Il est remarquable de constater à quel point cette alliance se fonde sur des liens personnalisés : " Nous avons une ingénieure de l'INCRA qui est une grande amie à nous. C'est grâce à elle que nous avons obtenu cet enseignement modulaire. »

Le recours à l'INCRA ne constitue toutefois pas une panacée, la question de l'écoulement des produits (cacao, wassaï, banane, pastèque, farine de manioc) demeurant entière en l'absence de transport. Malgré les pressions exercées, la voie d'accès à la route nationale, ébauchée sous le gouvernement Capiberibe (1995-2002), demeure sous le coup d'une suspension de l'IBAMA faute d'étude d'impact. Comme on l'a souvent observé, les institutions sollicitées enchaînent les initiatives sans véritable concertation ni calendrier. La construction d'une usine de transformation du cacao (pulpe et fève), inaugurée en mars 2007, sous le double parrainage du Ministère (fédéral) de l'Environnement et du Gouvernement de l'Etat d'Amapá, a ainsi précédé un énième programme d'assistance à l'association agroextractiviste du Cassiporé, assumé quant à lui par le Secrétariat d'Etat à 
l'Industrie, au Commerce et aux Mines (SEICOM) de l'Amapá. On ne saurait préjuger du résultat: lors de notre visite en septembre 2007, le nouveau président de l'association était absent, et l'ancien président, Sebastião, ne semblait guère optimiste.

Sous l'égide de l'INCRA, la communauté se sentit toutefois plus forte et mieux légitimée dans ses revendications. La légitimité repose avant tout sur l'occupation très ancienne, bien que celle-ci soit invoquée par des gens d'immigration récente (1950 pour la plupart), comme c'est le cas de Sebastião (" Voilà plus de trois cents ans que nous vivons avec la forêt, de génération en génération, et la forêt est intacte »), ou de D. Soeli Martins (" Nous autres, voilà plus de 400 ans que nous vivons sur le Cassiporé, nous sommes des enfants d'ici, nous le connaissons »). Sage gestion des ressources ? D’autres arguments dérivent des programmes de télévision, constamment invoqués à l'encontre des affirmations de l'IBAMA, perçues comme mensongères. On suggéra ainsi que l'IBAMA occultait le fait que ses ressources provenaient pour partie de pays étrangers, ces mêmes pays ignorant totalement - du fait de ces mensonges - que les zones protégées étaient peuplées d'humains. Les habitants de Vila Velha se sentent légitimés dans leur demande de compensation au point d'adopter une forme de chantage, ainsi de Dona Soeli qui réclame la création de 120 emplois publics en échange d'un arrêt de la chasse donnée localement au jaguar, espèce protégée. Sebastião Pinheiros exprime une idée similaire lorsqu'il affirme: "Bien sûr que le gouvernement ne peut pas donner un emploi à tout le monde, mais il doit faire en sorte que nous ne tombions pas dans l'erreur. (...) Nous savons que les forêts sont concentrées principalement ici, en Amazonie. Le monde entier veut la préservation, mais ceux qui vivent ici, et n'ont que leur emploi pour survivre à la sueur de leur front, si nous n'avons pas de terre pour travailler, qu'allons-nous faire?»

Ce discours de compensation a un modèle: celui du Président de la République Brésilienne, Luís Inácio da Silva dit Lula, rappelant aux pays « riches » leur responsabilité dans la dégradation de la planète, responsabilité qui se traduit par la demande de compensation pour le non-déboisement. Grâce à la télévision, ce discours trouve un écho favorable chez les populations traditionnelles d'Amazonie, et ce jusqu'aux Indiens qui s'identifient parfaitement à cette position du pauvre acculé à la misère par la cupidité des grands. L'idéal de " progrès » se dévoile, sous les paroles écologiquement correctes, dès que l'interlocuteur se sent atteint dans son aspiration à améliorer ses conditions d'existence. Pour Dona Soeli, certains dégâts écologiques sont une forme de "progrès ", tel le progressif ensablement du fleuve Cassiporé qui en diminua la profondeur et en ralentit le cours. De même, si la construction d'une voie d'accès à la BR156 est perçue comme une menace pour la sécurité des habitants, la possibilité de se procurer des biens de consommation prime, toujours selon Soeli, cet inconvénient.

Notre objectif, en citant ces extraits d'entretiens, était de montrer à quel point les habitants de Vila Velha exprimaient une position paradoxale quant à leur identité de "population traditionnelle», cultivant des arguments inattendus, mais traduisant au final une vision lucide, eût égard à leurs sources d'information, de leur situation et de leurs perspectives. L'oscillation permanente, due à l'interventionnisme extérieur, entre un investissement purement agricole et la recherche de ressources alternatives et écologiquement correctes ajoute probablement à la confusion générale, dès lors que l'adoption de principes de durabilité est conditionnée par un changement d'attitude de l'ensemble de la communauté. Les raisons de l'échec communautaire à s'agréger autour d'un projet commun sont perçues et exposées en toute clarté, certains habitants - les évangéliques ou crentes - refusant qui plus est de se soumettre aux injonctions d'une 
«traditionnalité » imposée, avec son cortège de coutumes et de liens solidaires. Ces raisons tiennent à l'histoire même de la communauté, à l'hétérogénéité de ses origines, de ses croyances, bref : elles résident dans l'identité de la collectivité elle-même.

\section{La communauté de São Francisco}

19 En juillet 2004, une mission d'expertise commanditée par le Fonds Français pour l'Environnement Mondial (FFEM) dans la Réserve de Développement Durable 5 de la rivière Iratapuru dressait un tableau assez sombre de la communauté de São Francisco, dont les outils de production d'huile de noix du Brésil (Bertholetia excelsa) et la fabrique de biscuits étaient partis en fumée, déchirée par des conflits internes, criblée de dettes, sollicitée de tous côtés par des représentants de l'Etat d'Amapá et de divers organes de recherche et d'ONG pour se prêter au jeu des consultations, des inventaires et des ateliers de formation (Pinton \& Lescure, 2004). Trois ans plus tard, en juin 2007, c'est un tout autre tableau que découvre la mission dépêchée par le projet DURAMAZ. Pour expliquer ce changement, on pourrait mentionner l'expulsion de la famille Castelo (dont l'aîné, Brás, contrôlait la coopérative) tenue pour fauteuse de troubles, un contrat avantageux avec l'entreprise Natura, ainsi qu'un partenariat consolidé avec la SEMA (Secrétariat de l'Etat d'Amapá à l'Environnement), alliance stratégique dérivant en une forme alternative de clientélisme (Araújo, 2004a). Ce ne sont là pourtant que des péripéties si l'on considère que les projets de Développement Durable dans la Réserve, qui reposent sur l'existence d'une coopérative, connaissent une certaine réussite en dépitde son mode de fonctionnement, et se perpétuent non pas en conservant l'homogénéité de la population de São Francisco, mais bien au contraire par l'absorption d'éléments extérieurs à celle-ci.

20 A l'inverse - et c'est notre première hypothèse - la principale source de dissension au sein de la communauté de São Francisco do Iratapuru est précisément la coopérative, du fait des intérêts financiers qui en dérivent, des contraintes qui pèsent sur les associés, et de l'amertume de ceux qui en sont exclus. Si la cohésion sociale est malgré tout préservée, c'est que la communauté présente des ressources intrinsèques permettant de contrebalancer les tendances centrifuges déclenchées par d'alléchantes perspectives économiques. Parce que la Coopérative mixte des producteurs et extractivistes du fleuve Iratapuru(COMARU, créée en 1992) constitue la seule institution organisée et représentative, à défaut de conseil communautaire, le mélange des genres et des intérêts crée des rancœurs chez ceux qui pour une raison ou une autre n'en font pas partie : « Tout ce qu'ils veulent, c'est contrôler, assujettir ", déclare d'emblée un jeune habitant d'arrivée récente, à qui l'on avait refusé un emploi dans la fabrique au motif qu'il n'était pas associé.

Mais parmi les fondateurs historiques tant de la communauté que de la coopérative on trouve également des positions radicales, comme c'est le cas chez Biló (Benedito Rodrigues), né approximativement en 1930, qui représente la troisième famille par ordre d'importance : «Celui du dehors qui a le plus aidé, c'est Brás [Castelo]. C'est lui qui a fondé cette coopérative. (...) A l'époque, tu travaillais, tu avais un déjeuner, un café, un goûter, et un dîner. On ne manquait de rien. Et aujourd'hui? Rien du tout. A l'époque, tu travaillais, tu étais payé. Aujourd'hui, on doit travailler en groupe : il n'y a ni café, ni déjeuner, ni dîner. Qui peut supporter ça? Tu reçois de l'argent, mais quand? Ceux qui ont décidé que Brás devait partir, c'est la famille de Sabá [Sebastião Freitas, actuel directeur de la COMARU] et Arraia [père de l'actuel président de la COMARU], ils ont pris le contrôle. » 
21

Suivent les traditionnelles accusations de corruption des dirigeants (argent détourné, chacun des membres de la coopérative ayant des moteurs hors-bords et les autres non), le fait essentiel étant, bien entendu, que ces dirigeants appartiennent aux deux autres familles, celle des Freitas (Sebastião) et celle des Viana (Arraia). La structure administrative de la coopérative déroge aux règles coutumières de gestion communautaire: Biló, extractiviste "à l'ancienne», préférait manifestement une direction extérieure qui plaçait les castanheiros (collecteurs de noix) en situation d'employé en régime d'aviamento(dette entretenue), situation qu'il connaît et dont il maîtrise les règles : « Tu travaillais, (...) tu avais le déjeuner, le café, le goûter, le dîner, on ne manquait de rien ». Dans le régime clientéliste, en effet, le patron assiste son employé et le récompense en nature: le salaire dédommage le travail; le café, le goûter, le déjeuner, quant à eux, signifient la relation paternaliste, le lien social privilégié. Loin de conforter la légitimité interne de la COMARU, l'expulsion manu militari de la famille Castelo n'a fait que susciter de nouvelles animosités, la source potentielle de conflit s'étant déplacée vers ce qui touche au ressort intime de la vie communautaire, l'équilibre entre les différentes familles qui la composent.

\subsection{La mémoire collective}

Pour comprendre cet équilibre, il nous faut remonter aux origines du peuplement actuel de l'Iratapuru, une vague d'immigrants venus de Breves, île de l'embouchure de l'Amazone, dont les premiers arrivés appelèrent leurs proches à les rejoindre. Le travail consistait alors en extraction diversifiée : latex d'hévéa (Hevea brasiliensis) et de balata ( Manilkara bidentata), et noix du Brésil, produits auxquels il faut ajouter les peaux de félin (jaguar et ocelot). L'implantation du projet démesuré du milliardaire américain Ludwig, dans les années 1970 (usine de cellulose, plantation d'arbres à croissance rapide, riziculture et élevage, et fondation des villes de Monte Dourado, Laranjal do Jari et Vitoria do Jari) n'affecta guère la vie des habitants de l'Iratapuru, qui vivaient éparpillés le long des affluents, proches de leurs abattis et de leur sites de noyers du Brésil (castanhais). C'est la nécessité de donner une éducation à leurs enfants qui emporta la décision de créer une agglomération à la confluence de l'Iratapuru et du Jari, actuelle bourgade de São Francisco. La communauté actuelle fut donc formée au début des années 1980, et la coopérative ne vint que par la suite, en 1992. Or cette agrégation ne fut rendue possible que parce que le sentiment d'une communauté de destin unissait ces habitants, qui se connaissaient, se fréquentaient, et avaient, pour la plupart, connu le même parcours.

La principale originalité de ce peuplement est d'avoir substitué une ancienne population de collecteurs de caoutchouc et de noix du Brésil soumis à l'autorité du « colonel » José Júlio de Andrade, propriétaire d'un domaine vaste comme la Belgique, qui dominait le Jari et tous ses affluents. Cette population a disparu sans laisser de traces, fermant ainsi une page sombre de l'histoire: les récits portant sur le «Temps du cep » témoignent de la généralisation des procédés employant la terreur pour soumettre les employés ou «clients». Les contremaîtres de José Júlio se chargeaient du transport régulier d'employés vers les sites d'extraction, pour les rapatrier à la fin de la saison, ainsi qu'en témoigne Zé Laranja: «Les gens travaillaient ici puis redescendaient. Ils n'y habitaient pas vraiment. [C'était] le Temps du cep (tempo do pau). Quand je suis arrivé, j'ai entendu dire que le caboclo venait travailler, il recevait une machette, et quand il descendait on l'attachait. On lui tapait dessus au lieu de le payer.»

Développement durable et territoires, Varia (2004-2010) 

désignation de "pau», l'emploi du cep (pièces de bois articulées entre lesquelles on enserrait les chevilles, les mains ou le cou des suppliciés), répandu dans toute la région amazonienne depuis la Colombie jusqu'aux Guyanes (les Indiens de l'Uaçá en ont restauré l'emploi tout récemment), supplice décrit par Michael Taussig (1987) dans son étude sur l'usage de la terreur à l'ère du caoutchouc. Biló nous a décrit de manière très vivante (quoique un peu obscure) la manière dont Zé Júlio procédait au paiement de ses employés, d'après, semble-t-il le témoignage de son père venu travailler une saison. Il confirme l'existence du cep, et relate des épisodes où des employés venus réclamer leur paye furent abattus à coup de revolver. Bien qu'il n'ait pu le connaître, le vieux Biló imite, lorsqu'il le cite, la voix rauque du colonel Zé Júlio, montrant ainsi qu'on lui en a fait une description plutôt vive.

Il est remarquable de constater à quel point les habitants actuels d'Iratapuru se sont appropriés l'histoire locale, véhiculée par d'anciens habitants, au point de s'inscrire dans sa continuité. Cette histoire a fait l'objet d'un processus de réélaboration dont nous avons cherché, ailleurs (Kohler, 2009), à démonter les mécanismes. Cette réélaboration, qui se fonde sur un ordre logique mais non chronologique, réunit en un seul épisode trois événements singuliers : la révolte d'un aviado (travailleur prisonnier de sa dette) nommé Cezário, en 1928; la disgrâce de Zé Júlio qui le mena à s'exiler durant quelques années à Funchal, île de Madère (1932); et enfin, la cession de ses activités au «consortium » dit « des Portugais », en 1948 (Lins, 1997 ; 2001). L'implantation dans un lieu historiquement «chargé » permet ainsi la constitution d'une mémoire collective et d'un monde de références communes, l'espace renvoyant intrinsèquement à la temporalité ; un moment du passé correspond en effet à un état de peuplement et d'occupation de l'espace, comme en témoigne Benedito Baía dit Piara : "En ce temps-là, ça commençait avec Basilio, plus bas venait Duca Fonseca, dans l'Estorana... Non, d'abord il y avait Salvador, en amont. Ensuite, le défunt Vavá, plus bas, à Bananeira (...). Puis à Chapéu Virado c'était Souza, Dico Souza qui est mort aussi (...). Partant de là c'était Castor à la bouche de l'Amazonas, qui habitait seul, puis Zé Laranja à São Francisco (...). Plus bas, il y avait Queiroz à Santa Rita, Pascoal à Bacaba, le défunt Cabôco à Abacate, et puis Manduca à São João, et Macário du côté de Limão... »

Mais il est d'autres histoires que se sont appropriés les habitants de l'Iratapuru : celles des antiques habitants et des créatures surnaturelles qui peuplent les forêts à l'entour, présentant semblable manière d'entremêler mémoire et localités - ce que Laura Rival (1993) appelle mémorialisation. C'est ce que suggère l'énumération que nous fit Biló, lorsque nous lui demandions de nous parler des visagens (apparitions) : "Qui sait combien de gens sont morts par ici. Les Indiens, ils habitaient plus bas, vers Samaúma. (...) A la première colocação (zone de collecte) de l'affluent Amazonas (...) on trouvait des fragments de céramique. Làbas, au Poção, on a trouvé un pain de manioc de cette taille. [Le Curupira, créature de la forêt] c'est un petit noir, un homme. On dit qu'il n'a pas de fesses. Il surgit (...) quand le temps est à l'orage. Au Centrão, c'est là qu'il habitait. "

Comme on peut le constater, les habitants de l'Iratapuru, malgré une arrivée relativement récente, ne semblent pas résider en un lieu vide de sens et d'histoire, dépourvu de profondeur temporelle. En cela, ils témoignent d'un ancrage dans la localité, condition nécessaire de la permanence. 


\subsection{Corésidence et parenté}

27 Plusieurs indices nous permettent de reconstituer la progressive substitution d'une population par une autre, entraînant l'appropriation d'une mémoire locale incluant la toponymie. D'une part, il semble clair que les seringueiros et castanheiros du temps de Zé Júlio, pour la plupart, ne résidaient pas sur place : ils étaient des saisonniers, prisonniers du régime de la dette, victimes de mauvais traitement, et l'on peut supposer que les équipes étaient renouvelées quasi annuellement. Il est vraisemblable que parmi ces équipes aient figuré des hommes originaires de Breves : Biló fait allusion à son père (qui dut probablement revenir à Breves après cette expérience), mais celui qui réellement créa la nouvelle souche de peuplement fut le dénommé Basílio, originaire de Gurupá, qui travaillait sous le régime de la dette pour le compte d'Orlando Barreto, résident de Cachoeira de Santo Antônio; selon Roberto Araújo (2004b), Basílio est «l'ancêtre commun » reconnu par les deux principales familles. Venu au début des années 1950 de Gurupá accompagné de ses filles, les immigrants suivant devinrent ses gendres, ou épousèrent ses petites-filles, comme c'est le cas d'Arraia (père de l'actuel président de la coopérative), tandis que le directeur actuel, Sebastião Freitas, est son petit-fils en lignée maternelle.

En d'autres termes, dès l'origine de ce nouveau peuplement, se met en place un système où s'établissent des rapports privilégiés entre un beau-père et ses gendres (notons que Biló demeura à l'écart de ce système). Il serait vain, sans doute, d'extrapoler ; mais si l'on songe que le rejet de la famille Pinheiros à Vila Velha do Cassiporé est dû au fait que cette famille comptait de nombreux fils, accaparant ainsi les femmes disponibles, on peut légitimement se demander si le hasard des sexes n'a pas joué un rôle dans l'établissement, à Iratapuru, d'un système de transmission d'héritage plus équilibré 6 .La famille du fondateur se scinde en autant de lots qu'il a de gendres, à la fois autonomes, fondateurs de nouvelles lignées, et reliés les uns aux autres par l'ascendance de leurs épouses respectives - ce fait aura de grandes conséquences pour la pérennité de l'extractivisme local, comme nous le verrons plus avant.

Cette double affinité - les nouveaux venus étaient tous originaires du canton de Breves et se trouvaient alliés du fait de leur mariage - explique en grande partie l'homogénéité actuelle de la communauté de São Francisco, et la relative harmonie qui y règne. Toutefois, cette même communauté possède des mécanismes permettant d'intégrer de nouveaux-venus ou des individus sans parenté directe, car comme on l'a souvent observé la corésidence crée une forme de parenté (Gow, 1991). C'est ce que Sebastião Freitas, directeur de la coopérative, exprime sans détour: "Aujourd'hui, ici dans la communauté, tout le monde est parent. Tout le monde ici est parent ou presque. Parfois d'un peu loin, mais parent. (...) Dona Maria Helena n'est pas notre parente. Mais nous la considérons comme une parente depuis le temps qu'elle vit avec nous. Alors on ne peut pas dire qu'elle ne l'est pas, nous la considérons comme notre tante."

Cette forme de parenté joue un rôle essentiel dans le réseau de solidarité qui structure la communauté, forme à laquelle viennent s'ajouter deux phénomènes couramment observés à Iratapuru: la circulation des enfants et l'hébergement permanent ou provisoire de non-apparentés. Nombre de familles en effet adoptent, provisoirement ou définitivement, des enfants consanguins ou non apparentés - enfants abandonnés, ou nés de femmes trop jeunes ou déjà trop pourvues ; quant aux non-apparentés (agregados), ils 
sont généralement des saisonniers qui, à cause de services rendus, résident à demeure comme des membres de la famille.

31 L'intégration à un réseau de parenté, si elle est une condition nécessaire, n'est pas suffisante toutefois à «faire communauté». En effet, le représentant de Vila Velha du Cassiporé, dont on a vu les dissensions internes, tient à peu près le même discours que Sebastião Freitas: "Pratiquement, nous sommes une seule famille; des 300 habitants, 95\% appartiennent à la même famille, et $5 \%$ sont des gens qui sont arrivés après, mais en pratique c'est $100 \%$ parce que les $5 \%$ qui sont venus d'ailleurs sont de la famille, ils font partie de la famille, ils se sont mariés, ils ont fondé une famille dans la communauté. "

Il faut donc aller chercher plus loin les ressorts de la solidarité qui se manifeste à São Francisco. On ne pourra éluder ici la question religieuse, dans la mesure où la conversion de certains habitants au protestantisme explique en grande partie les scissions de l'autre communauté étudiée, celle de Vila Velha du Cassiporé.

\subsection{Rôle du catholicisme}

32 Nous parlons en effet de communautés caboclas, c'est-à-dire de communautés issues de la déstructuration (ou désagrégation) du monde amérindien par la réduction en esclavage, les épidémies, et regroupées à des fins d'évangélisation. Selon Delaunay (1984), la diminution de la taille des groupes a entraîné une restructuration en noyaux familiaux, qui durent élaborer de nouvelles stratégies d'alliances afin de maintenir le seuil critique de main d'œuvre en deçà duquel il n'est plus d'horticulture possible. Ce rôle fut assigné à l'institution du baptême, qui revêt en Amérique catholique une importance fondamentale sous les formes du compadrio ("compérage »). Le lien qui unit deux « compères » va bien au-delà de la stricte éducation du filleul: le compadrio est un système d'échange privilégié entre deux familles n'ayant pas de lien de parenté, qui instaure ainsi une solidarité obligée, encadrée par le rituel du parrainage.

La perte d'influence du catholicisme après le Concile Vatican II et la répression des militaires sera compensée par les religieux engagés dans la Théologie de la Libération, branche marxiste de l'Eglise romaine qui réorganisera en profondeur les communautés traditionnelles en luttant à sa manière pour la réforme agraire. Cette réforme s'appuyait sur la formation de "communautés ecclésiales de base ", la communauté étant ainsi élevée au rang de catégorie politique car elles se voulaient à la fois des modèles inspirés des premiers temps de la Chrétienté dans un Brésil rural marqué par le latifundium hérité de l'époque coloniale. Ainsi furent créés nombre de syndicats paysans, grâce à la formation de représentants communautaires, dont tant Sebastião Freitas, d'Iratapuru, que Sebastião Pinheiros, de Vila Velha, sont des exemples achevés. Un lien étroit s'établit alors entre rituel religieux et organisation politique, la légitimité des représentants s'appuyant sur leur aura de leaders religieux. Là encore s'impose à nous la figure de Sebastião Freitas : «Pour former une communauté, voilà comment je vois les choses: il faut avoir une grande union (...), c'est le premier pas. Ensuite, ce qui est très important, c'est la partie religieuse. Mais pour cela il faut quelqu'un qui sache conduire la partie religieuse, sinon ça n'avance à rien. »

Or Sebastião Freitas fait justement partie de ceux qui suivirent une éducation religieuse, cela dès l'âge de 17 ans, dans la perspective de formation de leaders communautaires fomentée par les prêtres progressistes. Cette formation, dispensée sous forme de séminaires, incluait différents principes de gestion des conflits et d'organisation sociale. 
S'il devait exister une confusion entre communauté et coopérative, elle serait ici totale. En effet, il serait bien difficile de distinguer ce qui, dans le comportement de Sebastião Freitas, dérive de sa fonction de directeur de la COMARU, ou de son autorité morale; ainsi, lorsque Sebastião Freitas avoue un excès de boisson, c'est dans la plus parfaite confusion des rôles. Rabroué par le prêtre, qui lui rappelle sa position de "chef», il « promet que cela n'arrivera plus ».

Plus simplement, nous pourrions en déduire que la fonction de directeur est ancrée dans l'exemplarité, son autorité étant perçue comme morale : plutôt que de ses capacités de gestionnaire, Sebastião Freitas en a hérité du fait de son éthique, de son « respect » pour la communauté. Ce qui vaut pour l'homme vaut également pour les institutions locales. En effet, parallèlement à la consolidation de la COMARU, la communauté de São Francisco travaille à la revitalisation du culte de Saint François, en restaurant la chapelle, en faisant bénir l'image du saint par l'évêque de Macapá, achevant en cela de s'affranchir de leur origine insulaire, marquée par le culte de Saint Benoît de Gurupá. Le choix d'un saint, ainsi qu'on l'a souvent remarqué (Tassinari, 2003; Grossi, 2004), a donc une portée politique, dans la mesure où la puissance du saint permet de souder la collectivité tout en confortant le poids régional de la communauté qui l'abrite.

Si nous poursuivons à présent notre parallèle avec Vila Velha do Cassiporé, nous pourrions émettre l'hypothèse selon laquelle la multiplication des religions est facteur de désagrégation sociale. Cette hypothèse peut être toutefois nuancée, voire inversée : il se pourrait en effet que ces allégeances multiples soient précisément le signe ou la manifestation de dissensions préexistantes. A l'origine de ces dissensions, on trouvera sans doute un peuplement hétérogène, des divergences portant sur les choix économiques, une discontinuité des pratiques qu'une connaissance de l'histoire locale (une « micro histoire ») nous permettrait d'éclairer.

\subsection{Sociabilité en forêt}

Mais un autre élément doit entrer en ligne de compte : l'espace physique où se trouve insérée la communauté. Car cet espace partagé, fondement des références communes et de la mémoire collective, conditionne également la manière dont sont pratiquées les activités de collecte, selon le degré d'entraide exigé par la conformation des sites et les moyens par lesquels on peut y accéder.

Dans le cas de São Francisco, la répartition des sites de noyer du Brésil, les sauts qui ponctuent le parcours et qui rendent l'accès difficile, et enfin les pratiques associées au ramassage des noix ont favorisé le maintien d'une sociabilité ancrée dans les mœurs, ce qui perpétue, de fait, l'ancien mode de relation entre habitants de l'Iratapuru, foyers dispersés de peuplement avant l'agrégation en communauté.

La période de collecte (de mars à juin), comme nous avons pu le constater, est un moment privilégié de lien social. Le départ pour l'amont s'accompagne d'une certaine effervescence dans les préparatifs et d'un grand enthousiasme - période de chasse, de pêche, de vie au grand air, de tranquillité, etc. Les familles, ainsi que les gendres et les belles-filles partagent un campement favorisant la promiscuité durant deux à trois mois d'affilée. Il n'est pas d'autre distraction, en l'absence d'électricité, que les conversations vespérales et les visites d'autres collecteurs en route vers leurs sites plus éloignés. Ces visites sont perçues comme des distractions et nombre d'activités sont alors effectuées en commun; les jeunes enfants partagent leurs jeux avec des compagnons de leur âge ; les 
visiteurs se prêtent volontiers à un coup de main pour charger les noix ou pour coudre les sacs, réparer un moteur, rafistoler un abri, chasser et dépecer le gibier, pour repartir généralement après avoir passé la nuit.

Mais c'est surtout durant la montée puis la descente de la rivière Iratapuru que le fait de former communauté prend tout son sens. Le franchissement des sauts serait impossible si les équipages ne s'attendaient pas mutuellement, prêts à se porter secours. C'est ce qui permet à des familles réduites (parents jeunes, enfants en bas âge) d'accéder à leur site malgré la lourdeur des pirogues de transport: elles préviennent de leur départ, s'enquièrent des trajectoires des uns et des autres, et généralement attendent l'arrivée d'autres collecteurs dans les abris aménagés près des sauts et des rapides.

C'est donc l'activité même de collecte qui fortifie la solidarité communautaire, par les besoins qu'elle engendre, par la convivialité qu'elle autorise dans les campements en forêt. Etrangement, ces périodes de travail intense ressemblent à des moments de détente, de vacances. On ne saurait appréhender les liens qui unissent les habitants de la Réserve de Développement Durableen ne s'intéressant qu'à la bourgade de São Francisco et aux infrastructures qu'elle abrite. C'est paradoxalement au moment où les familles se dispersent dans leurs sites respectifs que sont ménagés des moments d'échanges privilégiés, des visites ponctuelles qui sont de fait indispensables à l'approvisionnement, au chargement et au transport de la récolte.

La pratique extractiviste est donc par elle-même facteur de cohésion: elle impose et multiplie les occasions de travail collectif, les «mutirões ». Dans le même temps, elle permet de perpétuer la mémoire d'un système ancien, lorsque l'habitat était dispersé, et que les familles se rencontraient lors des fêtes religieuses ou du transport des noix vers ce qui était le centre économique de la région, Cachoeira de Santo Antônio, où étaient situés les entrepôts des intermédiaires, avant la fondation de Monte Dourado et Laranjal do Jari. Cette sociabilité est selon nous un ressort essentiel de l'intégration des saisonniers, pour la raison simple que nombre d'entre eux sont appelés à devenir des gendres. Et c'est en forêt que les gendres commencent à "faire partie de la famille ", c'est-à-dire qu'ils s'approprient l'histoire locale et la mémoire collective par les récits de veillée, tout en peaufinant leur apprentissage du métier.

\subsection{Transmission des savoirs et pérennité du système}

Ce dernier aspect nous permettra de répondre à la question de la transmission des savoirs locaux, sur laquelle repose, en ultime instance, la pérennité du système. Plusieurs obstacles sont en effet posés à la participation des enfants au cycle de la noix du Brésil. La certification du processus (certification FSC, qui concerne environ 20 sites sur 36) prohibe le travail infantile et exige que les enfants soient scolarisés ; une fois pourvus d'un savoir conséquent, ces mêmes enfants se verront offrir d'autres opportunités professionnelles, contrecarrant ainsi l'idéal d'une perpétuation d'activité «inscrite dans les gènes ». Les garçons, qui seraient concernés par la succession de leur père, s'y refusent du fait de la pénibilité du travail, puisque leur niveau d'étude leur ouvre d'autres portes. La direction de la coopérative ne peut absorber à elle seule cette main-d'œuvre qualifiée, aussi les garçons ont-ils tendance à rester en ville et à s'y marier. Pourquoi les garçons en particulier? Pour la raison simple que les filles, également scolarisées, interrompent leurs études lorsqu'elles rencontrent, au bourg ou sur le site de collecte (où tout conspire 
à nouer des liaisons sentimentales), le saisonnier qui sera leur futur mari, pour en tomber enceintes presque fatalement.

Le départ des jeunes scolarisés, c'est-à-dire la mobilité et la déterritorialisation qui participent du monde globalisé, constitue un problème majeur pour l'avenir de la communauté, ce que le président de la coopérative, Eudimar Viana, exprime en pleine conscience : «Ici, à ce que je vois, les parents font un grand effort pour que leurs fils apprennent le métier mais beaucoup s'en vont et ne reviennent pas. Ils vont en ville, ils y restent, trouvent un emploi, ont une vie assez bonne, et pendant ce temps la communauté voit se déliter son histoire, sa culture, elle perd peu à peu son identité. »

Or le système lui-même génère une alternative, ainsi que nous le suggérions. Cette alternative consiste en la transmission des savoirs et des sites de père à fille, et donc au gendre, ou d'épouse au mari. En effet, nombreux sont les couples formés, à Iratapuru, par une femme veuve ou séparée et un saisonnier généralement plus jeune qu'elle. Ces derniers cas, pour être fréquents, ne constituent pas la règle, qui repose davantage sur le mariage d'une fille du propriétaire de site avec un de ses employés. Eudimar affirme précisément, au moment où il déplore l'ampleur du phénomène, qu'il est lui-même le fruit de ces alliances passées entre le propriétaire et ses saisonniers. Son père Arraia, étranger à la communauté, épousa ainsi, nous l'avons vu, une petite fille du fondateur Basílio ; son futur beau-père le recruta à Laranjal do Jari "au cours d'une beuverie ». Eudimar évoque ainsi nombre de saisonniers "devenus d'ici » par le mariage, tout en s'inquiétant de l'ampleur du phénomène, du fait du grand nombre d'adolescentes disponibles qui, « tôt ou tard, causeront un problème ».

Or il ne s'agit pas là d'un épiphénomène, mais bien d'un des piliers sur lesquels repose le système extractiviste à Iratapuru, ce dont Eudimar ne semble pas avoir pris toute la mesure lorsqu'il feint de déplorer cette situation. Ce pilier, ce sont les femmes. C'est toute l'organisation communautaire qui concourt, en effet, à ce qu'un tel résultat se produise, comme le suggère l'affirmation répétée d'Eudimar selon laquelle ces saisonniers «sont devenus d'ici " (viraram daqui), de par le système de parenté étendue aux corésidents que nous décrivions précédemment. Loin de constituer un "problème», les femmes sont en réalité, ici, le pivot de l'organisation et de la perpétuation de l'extractivisme. Car cette situation permet de résoudre le paradoxe que nous évoquions : si le système demeure, c'est précisément grâce au maintien d'un faible niveau de qualification. Le mécanisme mis en place aboutit à un renouvellement démographique par l'intégration et l'absorption des saisonniers, peu ou pas formés, dans le réseau communautaire.

\section{Conclusion}

Nous avons choisi pour illustrer notre propos deux communautés assez semblables, afin de mettre en évidence les mécanismes fragiles qui font que certains projets « durables ", à conditions égales, sont voués au succès ou à l'échec. Dans le cas de Vila Velha du Cassiporé, la communauté est menacée de dissolution par les conflits internes, souvent cristallisés autour de l'appartenance religieuse. Les stratégies d'évitement sont mises à mal par l'exigence de solidarité exprimée par les bailleurs de fonds. Les différentes Églises qui surgissent sont symptomatiques de la nécessité d'exprimer, en termes compatibles avec des formes de pensée stéréotypées, des divergences idéologiques informulées portant sur les modes de subsistance et de rapport au milieu social et naturel. A São Francisco, la divergence exprimée par le vieux Biló sur la gestion de la coopérative, qu'il 
formule en termes de corruption et d'accaparement du pouvoir, dérive en réalité du modèle intériorisé de l'aviamento (dette entretenue) dont il ne parvient pas à se défaire. Cette discordance est toutefois sans conséquence pour São Francisco car elle est minoritaire.

Le deuxième objectif, dans le cadre du projet DURAMAZ dont cet article est issu, était de placer en miroir les apports respectifs des approches socioéconomique et anthropologique. Nous avons donc posé deux hypothèses paradoxales que nous nous sommes efforcés de résoudre.Puisque la coopérative est la principale source de dissensions à São Francisco, le maintien de la cohésion sociale doit être cherché dans les formes d'organisation communautaires, dans la perception de ce qui fait communauté : une appropriation de la mémoire locale, un système de parenté élargie, une institution religieuse parallèle à l'administration de la coopérative, un modus operandi extractiviste qui favorise les travaux collectifs et donc la solidarité du groupe, et enfin un mécanisme de renouvellement démographique qui permet de compenser la rupture de transmission aux héritiers directs.

La deuxième hypothèse dérive d'un constat : l'un des obstacles majeurs à la pérennité du système extractiviste repose sur la difficulté de concilier une activité traditionnelle à faible valeur ajoutée avec une amélioration du niveau de vie et des perspectives due à l'augmentation du niveau d'études des jeunes générations. Dès lors, ou le système dérive vers un mode de gestion et de production modernisé, adapté aux compétences des futurs extractivistes - et le caractère «traditionnel » de l'activité devient pure fiction; ou - et c'est la solution qui s'est imposée d'elle-même à Iratapuru - la tradition est perpétuée par le maintien d'un faible niveau de qualification, grâce à l'intégration des saisonniers par le mariage, les femmes devenant dès lors détentrices du capital et permettant sa transmission.

\section{BIBLIOGRAPHIE}

ARAÚJO Roberto, 2004a, « Populations traditionnelles et développement durable dans des aires protégées en Amazonie : actions collectives ou clientélisme alternatif ? MPEG/MCT, ATI Aires protégées, IRD, Orléans.

ARAÚJO Roberto, 2004b, «Informações preliminares sobre a localidade de São Francisco do Iratapuru », rapport de mission, inédit.

BOYER Véronique (2009), La renaissance des perdants : Evangéliques, évangélistes et migrations, Paris, Karthala.

DELAUNAY Daniel, 1984, « Indien, caboclo et paysan : formation du paysannat dans le Ceará », Cahiers de l'ORSTOM, vol. XX n²1, p.43-69.

GALVÃo Eduardo, 1955, Santos e visagens - Um estudo da vida religiosa de Itá, Amazonas, São Paulo, Cia Editora Nacional. 
GOW Peter, 1991, Of mixed blood - Kinship and History in Peruvian Amazonia, Oxford, Clarendon Press.

GRENAND Pierre \& GRENAND Françoise, 1987, « La Côte d'Amapá, de la bouche de l'Amazone à la baie d'Oyapock, à travers la tradition orale palikur », Boletim do Museu Paraense Emílio Goeldi, sér. Antropologica, 3 (1), p.1-77.

GRENAND Pierre \& GRENAND Françoise, 1990 «L'identité insaisissable du caboclo amazonien », Etudes Rurales n¹20, oct-déc., p.17-39.

GROSSI Gabriele, 2004, Ici nous sommes tous parents, Fêtes et rituels chez les Pataxó du Mont Pascal, Thèse de Doctorat, EHESS.

KOHLER Florent, 2009, Tombeau des Aymorés: le monde souterrain des Indiens Pataxó, Paris, Louvain, éditions Peeters/SELAF.

GREISSING Anna, KOHLER Florent, LE TOURNEAU François-Michel, PICANCO José Reinaldo, 2008, « Iratapuru et la noix du Brésil : une expérience de durabilité en Amazonie brésilienne », Cybergeo , Environnement, Nature, Paysage, article 432, mis en ligne le 17 novembre 2008, URL : http:// www.cybergeo.eu/index20763.html.

LINS Cristóvão, 1997, A Jari e a Amazônia, Rio de Janeiro, Dataforma.

LINS Cristóvão, 2001 [1990], Jari, 70 anos de História, Rio de Janeiro, Dataforma.

PINTON, Florence, EMPERAIRE Laure, 1992, «L'Extractivisme en Amazonie brésilienne : un système en crise d'identité ", Cahier des Sciences Humaines 28 (4), p. 685-703.

PINTON Florence \& LESCURE Jean-paul, 2004, « Rapport de mission dans la Réserve de Développement Durable du Rio Iratapuru, Amapá, Brésil », projet financé par le Fonds Français pour l'Environnement Mondial (FFEM), inédit.

RIVAL Laura, 1993, «The Growth of Family Trees : Understanding the Huaorani perception of the Forest », Man, 28, p.636-652.

TASSINARI Antonella Maria Imperatriz, 2003, No Bom da Festa - O processo de construção cultural das Famílias Karipuna do Amapá, São Paulo, EdUsp.

TÖNNIES Ferdinand, 1977, Communauté et société, PUF (Gemeinschaft und Gesellschaft, 1887).

WEBER Max, 1971 [1922], Economie et Société, Plon.

\section{NOTES}

1. Ce travail a été réalisé dans le cadre du projet DURAMAZ - Déterminants de la durabilité en Amazonie brésilienne, financé par l'Agence Nationale de la Recherche et coordonné par Martine Droulers, directrice de recherche au CREDAL. Il est le résultat de différents terrains menés par des équipes pluridisciplinaires. François-Michel Le Tourneau, deux terrains de trois semaines: SIG, étude socio-économique. Florent Kohler, un terrain de trois semaines: entretiens biographiques non dirigés, enregistrés auprès de quinze informateurs. Anna Greissing, deux terrains totalisant six mois: entretiens non dirigés et application de 80 questionnaires biographiques et institutionnels portant sur les conditions socio-économiques, le niveau d'éducation, les histoires de vie, la sensibilité environnementale, et la perception locale du « développement durable».

2. L'usage en français du terme "extractivisme» et ses dérivés («extractiviste», «agroextractivisme »), qui désigne une activité de collecte de produits naturels renouvelables, 
est à juste titre contesté. S'agissant d'une publication à des fins de divulgation scientifique, toutefois, nous nous en tenons à cette désignation qui offre l'avantage d'être reconnue dans toutes les indexations.

3. Portaria/INCRA/SR(21)AP/G no 064 , de 27 de dezembro de 1999. Processo $\mathrm{n}^{\circ}$ : 54350.002755/99-48.

4. Source SEICOM: http://www.seicom.ap.gov.br/noticias/noticia5.html.

5. La RDS Iratapuru (« Reserva de desenvolvimento sustentável », créée par la loi nº392 du 11 décembre de 1997) est la plus grande unité de conservation de l'Etat d'Amapá. La catégorie de Réserve de développement durable fait partie du Système National des Unités de Conservation. Il s'agit d'unités qui abritent des populations traditionnelles dont l'existence repose sur l'exploitation durable des ressources naturelles. Leur objectif est de préserver la nature, mais d'assurer en même temps une amélioration des conditions de vie de ces populations et une valorisation de leurs connaissances sur la nature. Les activités autorisées ou non, ainsi que les relations entre les populations des réserves et les autorités doivent être régies par un plan de gestion.

6. Une remarque de l'actuel directeur de la COMARU va dans ce sens, lorsqu'il suggère que le pouvoir des Castelo (expulsés en 2002), et donc la menace qu'ils représentaient, venait du fait qu'ils étaient neuffrères.

7. Ce modèle explique en grande partie le fait que la « communauté » se pense à la fois comme un idéal à atteindre et comme un modèle fondé sur un âge d'or situé dans le passé. On entend ainsi déplorer souvent que la « communauté » ne soit plus ce qu'elle était, en se fondant sur un modèle qui n’a jamais existé dans la réalité.

\section{RÉSUMÉS}

Les populations traditionnelles de l'Amazonie brésilienne sont soumises, de la part des organes institutionnels et de l'opinion publique, à une double injonction : préserver la forêt tout en se pliant à des expériences de développement durable destinées à améliorer leurs conditions d'existence et leur formation. Deux études concomitantes menées en Amapá, l'une à Vila Velha do Cassiporé, l'autre à Vila de São Francisco do Iratapuru, nous permettent de dégager quelques principes conditionnant le succès ou l'échec des projets implantés. Ces principes concernent essentiellement la cohésion communautaire, et la disponibilité d'une main d'œuvre faiblement qualifiée, permettant ainsi le maintien d'activités qui pour être traditionnelles n'en sont pas moins pénibles.

The traditional populations of the Brazilian Amazon are subject to a double bind by institutional bodies and public opinion: these populations must preserve the forest while they act as subjects for sustainable development experiments intended to improve their living conditions and formal educational level. Two studies conducted in the State of Amapá, one in Vila Velha do Cassiporé, the other in Vila de São Francisco do Iratapuru; provide us with a few principles influencing the success or failure of these projects. These principles mainly concern community cohesion and the availability of an under-skilled workforce, which thus enable these populations to maintain activities that albeit traditional are nonetheless unpleasant. 


\section{INDEX}

Mots-clés : populations traditionnelles, extractivisme, réserve de développement durable, Amazonie brésilienne, communautés, communalisation, anthropologie

Keywords : Traditional populations, extractivism, sustainable development reserve, Brazilian Amazon, communities, communalization, Anthropology

\section{AUTEURS}

\section{FLORENT KOHLER}

Florent Kohler est anthropologue, maître de conférences à l'Université de Tours. Travaille sur les rapports homme/environnement, en particulier la question de la construction territoriale ( " géographie symbolique ») et des stéréotypes culturels à l'œuvre dans la formation des identités traditionnelles. florent.kohler[at]orange.fr

\section{ANNA GREISSING}

Anna Greissing est doctorante (EGIDE/Lavoisier), Centre de Recherche et de Documentation sur l'Amérique Latine, CREDAL, UMR 7169 du CNRS en cotutelle avec l'Institut de Géographie de l'Université d'Innsbruck. Thématiques de recherche: développement durable, politiques environnementales et stratégies de développement régional en Amazonie Brésilienne.

\section{FRANÇOIS-MICHEL LE TOURNEAU}

François-Michel Le Tourneau est géographe, chargé de recherche au CNRS, Centre de Recherche et de Documentation sur l'Amérique Latine, CREDAL, UMR 7169. Actuellement en poste au Centre de Développement Durable de l'Université de Brasilia (CDS-UnB). Spécialiste de la géographie de l'Amazonie brésilienne, il s'intéresse particulièrement à l'usage de l'espace chez les « populations traditionnelles ». 